BMC

Biochemistry

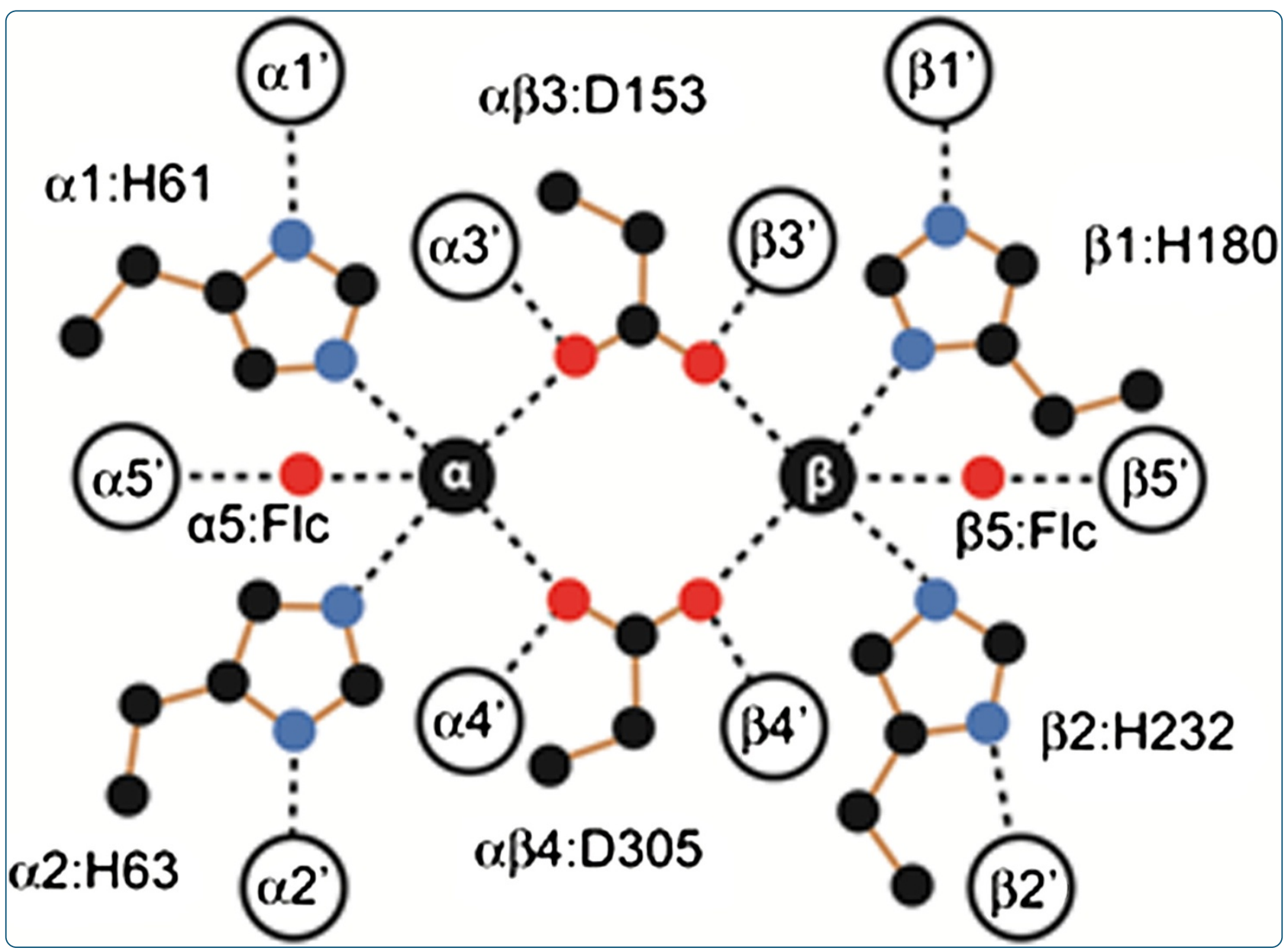

The mononuclear metal center of type-I dihydroorotase from aquifex aeolicus

Edwards et al.

() Biomed Central 


\title{
The mononuclear metal center of type-I dihydroorotase from aquifex aeolicus
}

\author{
Brian FP Edwards ${ }^{1}$, Roshini Fernando ${ }^{2}$, Philip D Martin ${ }^{1}$, Edward Grimley ${ }^{2}$, Melissa Cordes ${ }^{2}$, Asmita Vaishnav ${ }^{1}$, \\ Joseph S Brunzelle ${ }^{3}$, Hedeel Guy Evans ${ }^{1,2}$ and David R Evans ${ }^{1 *}$
}

\begin{abstract}
Background: Dihydroorotase $(\mathrm{DHO})$ is a zinc metalloenzyme, although the number of active site zinc ions has been controversial. E. coli DHO was initially thought to have a mononuclear metal center, but the subsequent X-ray structure clearly showed two zinc ions, a and $\beta$, at the catalytic site. Aquifex aeolicus DHO, is a dodecamer comprised of six DHO and six aspartate transcarbamoylase (ATC) subunits. The isolated DHO monomer, which lacks catalytic activity, has an intact a-site and conserved $\beta$-site ligands, but the geometry of the second metal binding site is completely disrupted. However, the putative $\beta$-site is restored when the complex with ATC is formed and DHO activity is regained. Nevertheless, the $\mathrm{X}$-ray structure of the complex revealed a single zinc ion at the active site. The structure of DHO from the pathogenic organism, S. aureus showed that it also has a single active site metal ion.
\end{abstract}

Results: Zinc analysis showed that the enzyme has one zinc/DHO subunit and the addition of excess metal ion did not stimulate catalytic activity, nor alter the kinetic parameters. The metal free apoenzyme was inactive, but the full activity was restored upon the addition of one equivalent of $\mathrm{Zn}^{2+}$ or $\mathrm{Co}^{2+}$. Moreover, deletion of the $\beta$-site by replacing the His 180 and His232 with alanine had no effect on catalysis in the presence or absence of excess zinc. The $2.2 \AA$ structure of the double mutant confirmed that the $\beta$-site was eliminated but that the active site remained otherwise intact.

Conclusions: Thus, kinetically competent A. aeolicus DHO has a mononuclear metal center. In contrast, elimination of the putative second metal binding site in amidohydrolyases with a binuclear metal center, resulted in the abolition of catalytic activity. The number of active site metal ions may be a consideration in the design of inhibitors that selectively target either the mononuclear or binuclear enzymes.

Keywords: Aspartate transcarbamoylase, Carbamoyl phosphate synthetase, CAD, Dihydrorotase, Metalloenzymes, Pyrimidine biosynthesis, Thermophile, Zinc ligands

\section{Background}

Dihydroorotase (DHO) catalyzes the reversible interconversion of carbamoyl aspartate and dihydroorotate, the third step in de novo pyrimidine biosynthesis. The protein is a member of the amidohydrolase family of enzymes [1] Figure 1.

The same reaction occurs in nearly all organisms but the enzymes are very diverse. A phylogenetic analysis [2] demonstrated that DHOs belong to two distinct classes that differ in sequence, size and oligomeric structure. Type-I enzymes, which evolved first, are larger, 43-45 kDa, and are often associated with aspartate transcarbamoylase (ATC),

\footnotetext{
* Correspondence: drevans@med.wayne.edu

'Department of Biochemistry and Molecular Biology, Wayne State University School of Medicine, 540 East Canfield Street, Detroit, MI 48201, USA

Full list of author information is available at the end of the article
}

the enzyme that catalyzes the formation of carbamoyl aspartate, the substrate for DHO. Type-II DHOs, which evolved more recently, contain only the catalytic core (38 $\mathrm{kDa}$ ) and typically have 50 and 10 residues deleted from the amino and carboxyl ends, respectively, as well as several internal insertions and deletions compared to the type-I enzymes.

E. coli $\mathrm{DHO}$, a type-II enzyme, is an eight stranded $\beta$ - or TIM barrel, a structural organization typical of amidohydrolyases [3]. While this enzyme was initially reported to have a mononuclear metal center [4], the X-ray structure showed that there are two zinc ions at the active site (Figure 2A) bridged by a water molecule and a carboxylated lysine (Lys 102). The zinc in the $\alpha$-site has a distorted trigonal bipyramidal coordination sphere and binds two histidines, an aspartate, the carboxylated lysine and the

\section{Biomed Central}




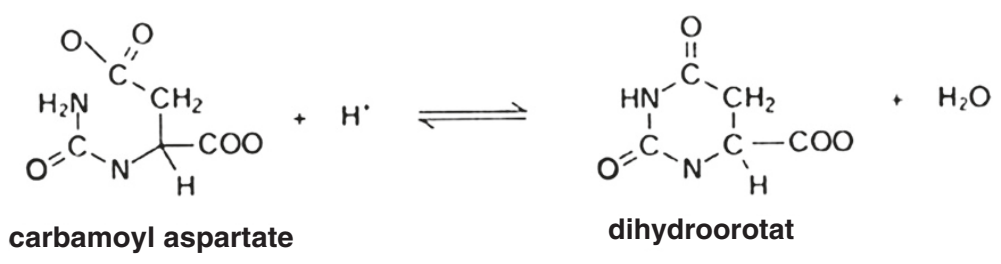

Figure 1 The reaction catalyzed by DHO.

bridging solvent. The zinc in the $\beta$-site is more solvent accessible and has a distorted tetrahedral coordination sphere consisting of two histidines, the carboxylated lysine and the bridging hydroxide. A catalytic mechanism was proposed based on the presence of two zinc ions and the juxtaposition of residues near the bound substrates [3].

We have cloned and expressed Aquifex aeolicus DHO, a type-I enzyme in $E$. coli $[5,6]$. The recombinant protein, with a molecular mass of $43 \mathrm{kDa}$, was expressed at high levels and was soluble but lacked catalytic activity. The enzyme was monomeric and contained a single zinc ion. However, it formed an active complex with $A$. aeolicus ATC consisting of six copies of each type of subunit that had both ATC and DHO activities [6]. The structure of the isolated DHO subunit, determined in two space groups is comprised of two domains, a distorted TIM barrel that resembles the structure of $E$. coli $\mathrm{DHO}$ and a composite domain formed by residues $1-55$ at the amino end and 366-422 at the carboxyl end of the polypeptide [7] that is found in many metal-dependent hydrolases [8-11].

The two A. aeolicus DHO structures suggested several possibilities that could account for the lack of activity of the isolated subunit. A water molecule was bound to the zinc in one X-ray structure (1xrf.pdb), while in the other structure (1xrt.pdb), Cys181 in a loop, designated loop A, was ligated to the active site zinc, occluding the active site and blocking access of the substrates. Most of loop A was disordered and invisible in the electron density map, as were two other loops, B and C. The zinc ion was bound to a site corresponding to the less solvent accessible $\alpha$-site in the $E$. coli enzyme. The zinc ligands were His61, His63, Asp153, and (Ja5) or Cys181 in an approximately tetrahedral arrangement (Figure 2A). The equivalent ligands for the zinc $\beta$-site in $E$. coli $\mathrm{DHO}$ were conserved but disarranged. Asp153 was close to the analogous carboxylysine in E. coli DHO but the two equivalent histidine ligands, His180 ND1 and His232 NE2 (A. aeolicus numbering) were rotated out of the active site by $7.4 \AA$ and $7.1 \AA$, respectively, from the putative zinc $\beta$-position.

The X-ray structure of the active A. aeolicus DHOATC complex (3d6n.pdb) showed that all of the disordered loops now had a well-defined conformation and that an extensive movement of loop A had displaced it from the active site [12]. The zinc in the $\alpha$-site now had tetrahedral geometry with His61, His63, Asp153, and one oxygen from citrate, a substrate analog, as ligands, with an average bond distance of $2.2 \AA$. The next closest ligand for trigonal bipyramidal coordination was Asp305 at $2.7 \AA$. Moreover, the $\beta$-zinc binding site was fully reconstituted when the DHO and ATC subunits associate to form the active dodecamer. However, no zinc was bound to the putative $\beta$-site, instead a water molecule, $\mathrm{HOH} 577$, (J $\beta$ in Figure 2A and B) occupied this position. As a consequence, one of the carbonyl oxygens of Asp153 that was formerly bound to the N of Gly154 now formed a hydrogen bond $(2.6 \AA)$ with the water molecule in the putative $\beta$-site. In addition, His180 and His232 rotate into the active site forming tight hydrogen bonds (His180 ND1: $2.2 \AA$; His232 NE2: $2.4 \AA$ ) with the same water molecule upon formation of the DHO-ATC complex (Figure 2A and B). Consequently, the positions of the zinc ligands were virtually the same as the corresponding $\beta$ zinc ligands in the E. coli enzyme (Figure 2C).

In mammals, dihydroorotase is a domain of a large multifunctional protein called CAD that also catalyzes the ATC and glutamine dependent carbamoyl phosphate synthetase activities $[13,14]$. It was previously found that mammalian DHO has a single active site zinc [15-17]. However, recent structural studies carried out by Santiago Ramon-Maiques (Spanish National Cancer Research Centre (CNIO), Madrid, Spain) showed that the DHO domain of human CAD, like the $E$. coli enzyme, has two zincs at the active site $\mathrm{a}^{\mathrm{a}}$.

Several observations have led many investigators to believe that the active site of all DHOs are binuclear, 1) the $\beta-\mathrm{Zn}$ site ligands are universally conserved, 2) these residues are located in the correct position (as in A. aeolicus $\mathrm{DHO}$ ) with an appropriate orientation to bind a second zinc, 3) the $\beta$-site is significantly more accessible to solvent, so that the second zinc may have been lost during protein purification or crystallization, 4) zinc analysis was typically preceded by dialysis to remove adventitiously bound metal $[4,14,15]$ that may have resulted in the loss of the second metal ion and 5) both E. coli and mammalian $\mathrm{DHO}$, which were initially found to have one zinc, were subsequently shown to have two metal ions.

The current study was undertaken to determine whether the lack of a second metal ion in A. aeolicus DHO is an 

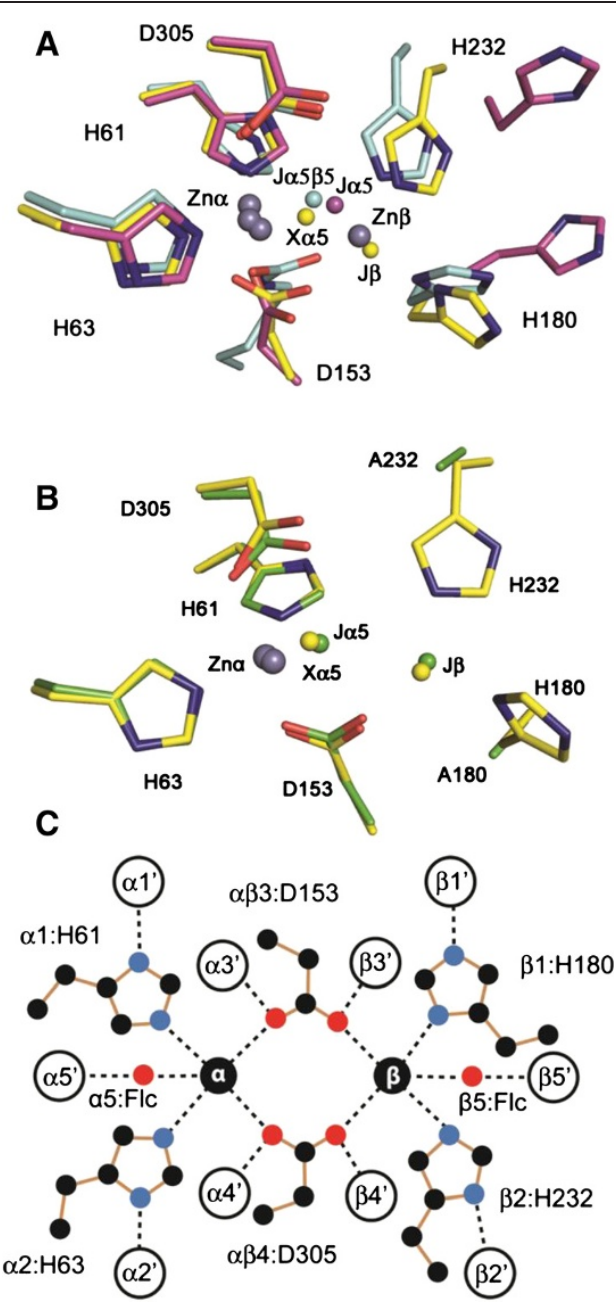

Figure 2 The DHO Active Sites. (A) The active sites of DHO from $E$. coli (pdb:1j79A; cyan carbon atoms), A. aeolicus (pdb:1 xrfA; magenta carbon atoms), and the A. aeolicus native complex of DHO and ATC (pdb:3d6nA; yellow carbon atoms). Zinc atoms are shown as grey spheres. Significant water molecules ("J") and ligand atoms ("X") are also differentiated by the carbon color. The water molecules are labeled by their role in the active site (Figure $2 \mathrm{C}$ ) because the numbers assigned to water molecules in the same site vary among the numerous DHO structures. Here, Ja5 35 is $\mathrm{HOH} 1407$ in 1j79.pdb; Ja5 is $\mathrm{HOH} 2$ in 1 xrf.pdb; $\mathrm{Xa} 5$ is the $\mathrm{OG} 1$ atom from the citrate ligand in $3 \mathrm{~d} 6 \mathrm{n}$. $\mathrm{pdb}$; and $\mathrm{J} \beta$ is HOH577 in 3d6n.pdb; (B) Superposition of the active sites of A. aeolicus DHO from the native complex (3d6n.pdb); yellow carbon atoms) and the mutant complex (4bjh.pdb; green carbon atoms). Zinc atoms are shown as grey spheres. Significant water molecules ("J") and ligand atoms (" $X$ ") are differentiated by the carbon color. Ja5 is HOH2018/A in 4bjh.pdb; Xa5 is the OG1 atom from the citrate ligand in 3d6n.pdb; and J $\beta$ is $\mathrm{HOH} 577$ in the wild-type $3 \mathrm{~d} 6 \mathrm{n}$. pdb and HOH2080/A in 4bjh.pdb. (C) A schematic diagram of the first and second binding shells for two zinc atoms in the active sites of type-l and type-II DHO enzymes. The first shell ligands are shown as labeled residues for A. aeolicus DHO. The second shell ligands are shown as labeled circles. The analogous first shell residues for S. aureus, B. anthracis, and T. thermophilus $\mathrm{DHO}$ and the second shell ligands for all four species are listed in the supplement (Additional file 1: Table S1) together with values for the distances marked by dashed lines in the diagram. artifact or whether the reversible condensation of carbamoyl aspartate to form dihydroorotate can be catalyzed by a mononuclear metal center.

\section{Results}

Metal analysis of the DHO-ATC complex and subunits

Zinc analysis by ICP-mass spectrometry confirmed the results of previous studies $[6,7]$ that indicated that the purified, isolated DHO subunit has only one mole of zinc per mole of protein (Table 1). The analysis also showed that the protein had no detectable bound cobalt, another metal ion that can support DHO catalysis. The growth media for the over-expression of ATC and DHO subunits was usually not supplemented with metals, so one possibility is that the $\beta$-site may have been unoccupied because the availability of the metal ion was limiting. To test this possibility, the growth media was supplemented with $100 \mu \mathrm{M}$ or $1 \mathrm{mM} \mathrm{ZnCl} 2$. The protein isolated from these different growth media (Table 1) also had one zinc atom and no cobalt, indicating that the unsupplemented media contained sufficient metal ions to saturate the protein metal binding sites. Similarly, the addition of $\mathrm{Zn}^{2+}$ or $\mathrm{Co}^{2+}$ to the purified DHO subunit did not significantly increase the incorporation of either metal ion (Table 2).

The zinc content of the DHO subunit and the DHOATC complex was also determined by a colorimetric 4-(2Pyridylazo) resorcinol (PAR) assay (Methods). The analysis (Table 3) was conducted at $25^{\circ} \mathrm{C}$, the temperature at which the crystals were grown, and at $75^{\circ} \mathrm{C}$, the temperature at which the enzymes were assayed. Analysis of the ATC subunit showed that it contained no detectable zinc. The DHO subunit had about 0.84 equivalents of zinc at $25^{\circ} \mathrm{C}$ and $75^{\circ} \mathrm{C}$ indicating that the metal binding $\alpha$-site was not completely occupied. The DHO subunit was then incubated with a 5-fold stoichiometric excess zinc, followed by removal of the excess metal by spin column chromatography. Zinc analysis indicated that the zinc content increased to 0.97-1.07, consistent with the interpretation that there was a small loss of zinc during the purification of protein. The presence of EDTA did not significantly alter the zinc content of the isolated DHO subunit.

A similar series of experiments was carried out on the DHO-ATC complex. This analysis (Table 3) indicated that the protein contained slightly less than one equivalent of

Table 1 Metal analysis ${ }^{\mathrm{a}}$ of DHO expressed with media supplemented with metal ions

\begin{tabular}{cccc}
\hline Protein & $\begin{array}{c}\text { Growth Media } \\
\mathrm{ZnCl}_{2}(\mathbf{m M})\end{array}$ & \multicolumn{2}{c}{ Molar ratio $\left(\mathbf{M}^{2+} /\right.$ protein $)$} \\
\cline { 2 - 4 } & 0 & $\mathbf{Z n}^{2+}$ & $\mathbf{C o}^{2+}$ \\
\hline $\mathrm{DHO}$ & 0.10 & 1.21 & 0.001 \\
& 1.00 & 0.97 & 0.000 \\
& & & 0.000 \\
\hline
\end{tabular}

${ }^{\mathrm{a}} \mathrm{ICP}$ mass spectrometry. 
Table 2 Metal analysis ${ }^{\text {a }}$ of purified DHO incubated with metal ions

\begin{tabular}{lcccc}
\hline Protein & $\begin{array}{c}\mathbf{Z n C l}_{2} \\
\mathbf{m M}\end{array}$ & $\begin{array}{c}\mathrm{CoCl}_{2} \\
\mathbf{m M}\end{array}$ & $\begin{array}{c}\text { Molar ratio } \\
\mathbf{Z n}^{2+}\end{array}$ & $\begin{array}{c}\left(\mathbf{M}^{2+} / \text { protein }\right) \\
\mathbf{C o}^{2+}\end{array}$ \\
\hline $\mathrm{DHO}$ & 0 & 0 & 0.88 & 0.001 \\
& 0.10 & 0 & 1.21 & 0.000 \\
& 1.00 & 0 & 0.97 & 0.000 \\
& 0 & 0.10 & 1.15 & 0.123 \\
\hline
\end{tabular}

${ }^{\mathrm{a}} \mathrm{CP}$ mass spectrometry.

zinc per DHO subunit, a value that increased to give approximately one-to-one stoichiometry when excess zinc was provided. Again, the zinc content was not appreciably altered by the addition of EDTA.

\section{Titration of DHO-ATC complex with zinc and cobalt}

To determine whether additional exogenous metal ions had an effect on the catalytic activity of the DHO-ATC, the complex was titrated with metal ions (Figure 3). There was a small activation of the DHO, approximately $20 \%$, as the molar ratio of zinc ion to protein increased, a further indication that the $\alpha$-site was not fully occupied. A similar result was obtained when the complex was titrated with cobalt chloride.

The endogenous zinc ion was extracted from the DHO subunit by exhaustive dialysis against the strong chelating agent, pyridine-2, 6-dicarboxylate (Methods). The complete removal of the metal was confirmed by zinc analysis. The metal free apoenzyme had no catalytic activity and was then titrated with zinc chloride and cobalt chloride. The

Table 3 Zinc analysis ${ }^{a}$ of wild type and mutant proteins

\begin{tabular}{lcccc}
\hline Protein & EDTA & Zn & \multicolumn{2}{c}{ Zn/DHO } \\
\hline ATC & $\mu M$ & molar ratio & $25^{\circ} \mathrm{C}$ & $75^{\circ} \mathrm{C}$ \\
Wild Type DHO & 0 & 0 & 0 & 0 \\
DHO & 0 & & & \\
DHO & 0 & $5 X$ & 0.84 & 0.83 \\
DHO & 100 & $5 X$ & 1.07 & 0.97 \\
DHO-ATC & 0 & 0 & 1.96 & 1.01 \\
DHO-ATC & 0 & $5 X$ & 0.98 & 0.95 \\
DHO-ATC & 100 & $5 X$ & 0.95 & 0.98 \\
DHO Double Mutant & 0 & & & \\
DHO & 0 & 0 & 0.71 & 0.90 \\
DHO & 100 & $5 X$ & 0.94 & 1.27 \\
DHO & 0 & 0 & 0.98 & 1.30 \\
DHO-ATC & 0 & $5 X$ & 1.20 & nd \\
DHO-ATC & 100 & $5 X$ & 0.93 & 1.14 \\
DHO-ATC & & & 0.95 & 1.29 \\
\hline
\end{tabular}

${ }^{\mathrm{a}}$ Spectrophotometric analysis.

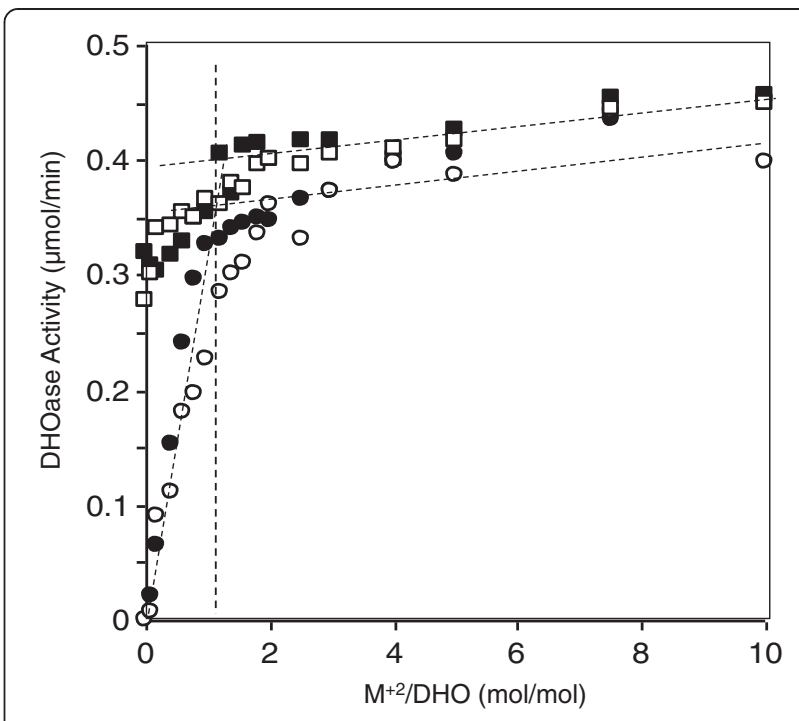

Figure 3 Titration of DHO-ATC and apo-DHO-ATC with zinc and cobalt. The DHO-ATC complex in 0.05 mM TrisHCl, pH 7.4 was titrated with zinc chloride ( $\square$ ) and with cobalt chloride ( $\square$ ) and the catalytic activity was assayed with a dihydroorotate concentration of $8 \mathrm{mM}$ at $72^{\circ} \mathrm{C}$. The metal ion was removed from the $\mathrm{DHO}$ subunit by dialysis against pyridine-2, 6-dicarboxylate and the DHO-ATC complex was reconstituted (Methods). The apoezyme was then titrated with zinc $(\bullet)$ and with cobalt ( $\square$ ) and assayed.

endpoint with both metal ions corresponded to a molar ratio of the metal ion to protein of one. There was only a slight increase in catalytic activity with increasing in zinc beyond the endpoint of the titration curve. The titration curves for zinc chloride and cobalt chloride were almost identical, indicating that both metal ions are equally effective in promoting catalysis.

\section{Effect of excess zinc on the steady state kinetics of DHO-ATC}

Although additional zinc had no apparent effect on the activity at near saturating concentrations of the substrate, excess metal ion may alter the steady state kinetic parameters. The dihydroorotate saturation curve for the DHOATC complex at $75^{\circ} \mathrm{C}$ (Figure 4, Table 4) gave $K_{m}$ value of $1.52 \pm 0.30 \mathrm{mM}$ and $a V_{\max }$ of $0.250 \pm 0.017 \mu \mathrm{mol} / \mathrm{min}$. The corresponding values for the complex assayed in the presence of a 5-fold molar excess of zinc gave $K_{m}$ and $\mathrm{V}_{\max }$ values of $1.93 \pm 0.35 \mathrm{mM}$ and $0.270 \pm 0.018 \mu \mathrm{mol} /$ min, respectively. Thus, there was little effect of additional metal ions on DHO catalysis or substrate binding.

\section{$\mathrm{X}$-ray structure of the $\beta$-site mutants ${ }^{\mathrm{b}}$}

Two residues, His180 and His232, that bind the second zinc ion in the $\beta$-site (Figure 2) of type-I DHO (T. thermophilus, 2z00.pdb; B. anthracis, 3mpg.pdb) and type-II enzymes (E. coli, 1j79.pdb; C. jejuni (3pnu.pdb); S. tymphurium, 3jze.pdb) and interact with a water molecule in A. aeolicus 
DHO (3d6n.pdb), were replaced with alanine by sitedirected mutagenesis. The single and the double His180Ala/ His232Ala were constructed. The double mutant was crystallized under similar conditions as the wild type enzyme [12] except that citrate, which binds weakly to the DHO and ATC active sites, was omitted. Both the DHO substrate, dihydroorotate, and the ATC bisubstrate analogue, PALA, were included in the "cryo" solution in an attempt to increase the order and thus the X-ray diffraction of the crystals. However, after preliminary refinements at higher resolutions, the data were truncated to $2.2 \AA, 0.1 \AA$ better than the native complex, at a conservative value of 2.3 for $\mathrm{I} / \sigma$ in the last shell. The final R-factor, using TLS refinement, is 0.159 and the final $R_{\text {free }}$ is 0.201 .

The asymmetric unit in space group $\mathrm{H} 23$ includes one DHO subunit with one zinc atom and dihydroorotate in its active site, one ATC subunit with PALA in its active site, three barium atoms bound to the ATC subunit, and twelve ethylene glycol molecules (PDB ID: edo). The barium ions, which increase the reproducibility, size, and diffraction of the crystals, have anomalous difference density peaks at 25, 15 and 7.0 sigma and are bound to the ATC subunit, which makes the sole crystal contact in the unit cell. The barium ions are quickly lost if omitted from the "cryo" solution, as was done with the native structure [12]. In this structure (Figure 5), the biological unit in the crystal is the same as the native structure, a tightly bound dodecamer with 23 symmetry formed by six asymmetric units. The full set of X-ray structure parameters are given in Table 5. The crystal structure of the double mutant showed strong anomalous difference density for zinc at the alpha site (above 17 sigma) and none at the beta site at the $3 \sigma$ contour level (Figure 6). Consequently, Zn423 at the alpha site was refined at full occupancy and a water molecule assigned to the A-

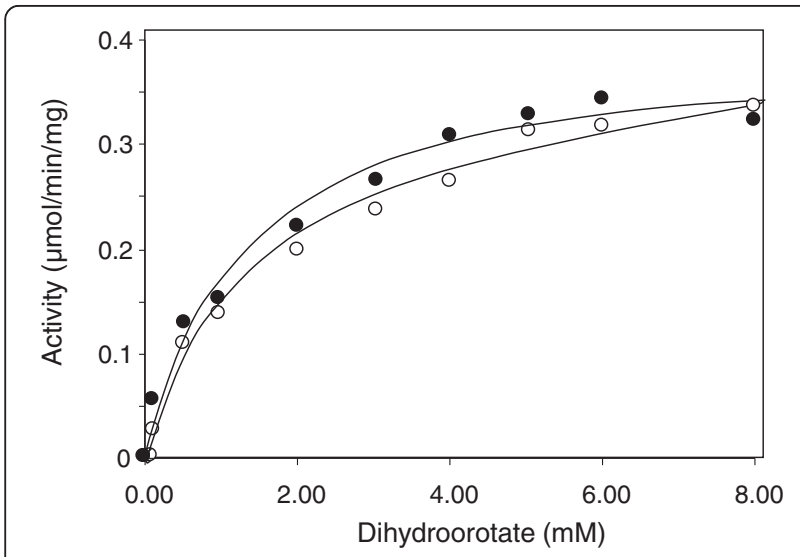

Figure 4 Effect of excess zinc on the DHO-ATC steady state kinetics. A dihydroorotate saturation curve of DHO-ATC (Methods; $250 \mathrm{mM}$ Tris acetate, $\mathrm{pH} 8.3,72^{\circ} \mathrm{C}$ ) was carried out in the presence of a 5 -fold molar excess of $\mathrm{ZnCl}_{2}$ (o) or in the absence of exogenous zinc $(\bullet)$. The kinetic parameters are summarized in Table 4.
Table 4 Steady state kinetic parameters of the wild type and mutant proteins ${ }^{a}$

\begin{tabular}{lcc}
\hline Protein & $\mathbf{K}_{\mathbf{m}}$ & $\mathbf{V}_{\max }$ \\
\hline Wild Type & $\mathrm{mM}$ & $\mu \mathrm{mol} / \mathrm{min}$ \\
Wild Type +5-fold $\mathrm{ZnCl}_{2}$ & $1.52 \pm 0.30$ & $0.250 \pm 0.017$ \\
Wild Type & $1.93 \pm 0.35$ & $0.270 \pm 0.018$ \\
His180Ala & $1.03 \pm 0.16$ & $0.233 \pm 0.011$ \\
His232Ala & $1.65 \pm 0.26$ & $0.238 \pm 0.013$ \\
His180Ala/His232Ala & $1.34 \pm 0.22$ & $0.224 \pm 0.012$ \\
\hline
\end{tabular}

${ }^{a}$ The dihydroorotate saturation curves (Figures 4 and 7) were fit by a least squares analysis to the Michaelis Menten equation using the program KaleidaGraph.

chain, $\mathrm{HOH} 2080 / \mathrm{A}$, was placed at the putative zinc beta site $(J \beta$ in Figure $2 B$ and Figure 6).

\section{Properties of the $\beta$-site mutants}

In the absence of exogenous zinc, the stoichiometry of metal binding to the double mutant (Table 3) exhibited somewhat less than one to one stoichiometry. The molar ratio of $\mathrm{Zn} /$ protein was $0.71-0.90$ for both the isolated DHO subunit and the DHO-ATC complex. As observed with the wild type enzyme, the occupancy increased to approximately $100 \%$ in the presence of a 5 -fold molar excess of zinc. The zinc titration of the His180Ala and His232Ala mutants resembled that of the wild type enzyme in that the catalytic activity increased approximately $20 \%$ in the presence of exogenous zinc, presumably the result of filling the incompletely occupied $\sigma$-zinc site. In contrast, there was only a modest increase in the activity of the double mutant with increasing exogenous zinc.

Dihydroorotate saturation curves of the DHO-ATC complex reconstituted with the DHO mutants and native ATC (Figure 7) indicated that the steady state kinetic parameters (Table 4) of the wild type, His180Ala and His232Ala mutants were very similar. The $K_{m}$ of the double mutant was unaltered (Table 4) but there was a small (21\%) but significant decrease in the $\mathrm{V}_{\text {max }}$.

\section{Discussion}

Metal analysis, the X-ray structure $[6,7,12]$ and anomalous scattering difference maps showed that the isolated, inactive A. aeolicus DHO subunit has a single, functional zinc-binding site. However, the extensive reorganization of the DHO structure that occurs upon formation of a complex with ATC, results in the generation of a catalytically active protein with an intact putative second metal binding site (Figure 2). Thus, the possibility that a zinc ion was lost during crystallization raises the question that a second, more loosely bound zinc ion could be required for activity. The initial metal analysis of E. coli and human DHO revealed a single active site metal ion, 


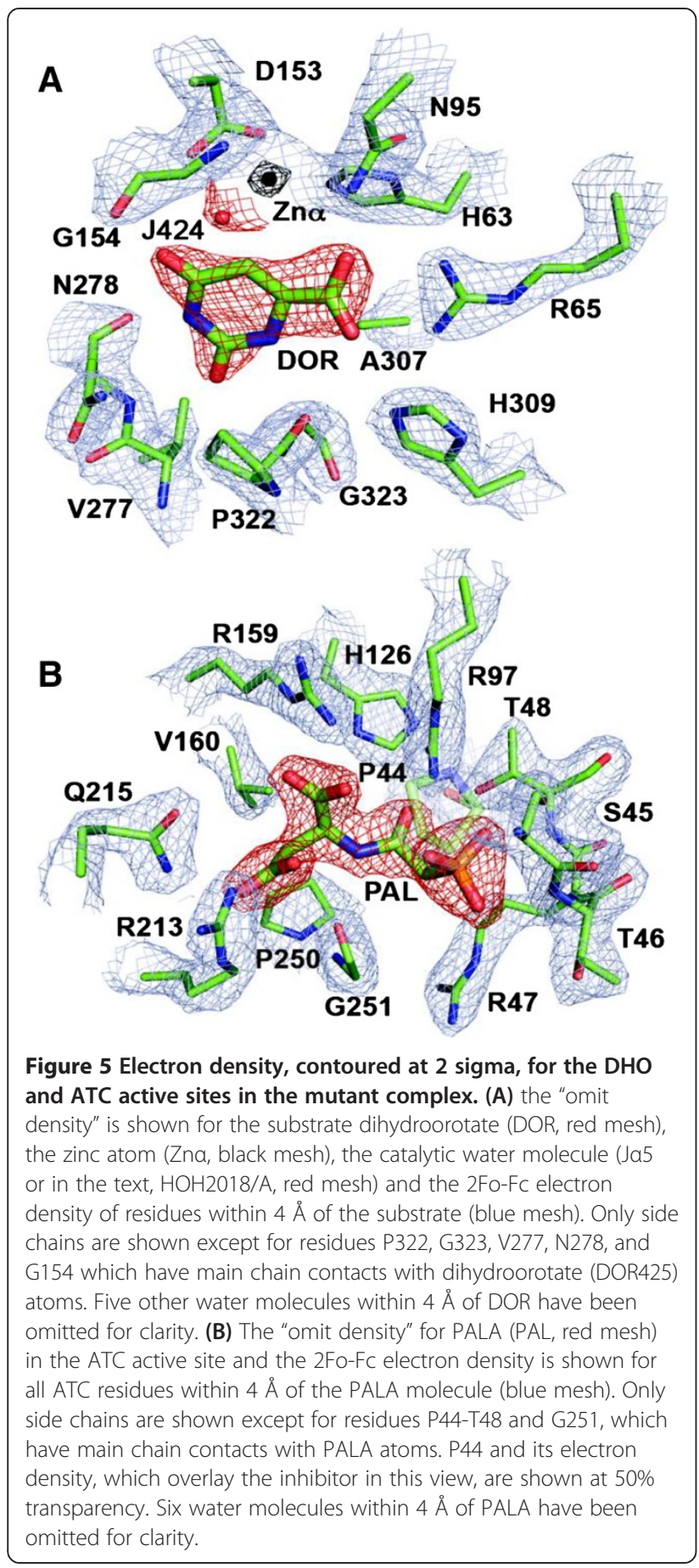

but both enzymes were subsequently found to have a binuclear metal center, an observation that lends credence to this hypothesis. The consensus is emerging that all dihydroorotases are binuclear. The distinction could be important as different strategies could be envisioned in the design of drugs that target DHO depending on whether the active site has one or two metal ions. Differences in the metal
Table 5 Crystal structure statistics

\begin{tabular}{lc}
\hline Statistics & Mutant \\
\hline PDB code & $4 \mathrm{BJH}$ \\
Crystals & 1 \\
Space group & $\mathrm{H} 32$ \\
Unit cell dimensions & \\
a $(\AA)$ & 157.15 \\
b $(\AA)$ & 157.15 \\
C (Å) & 233.24 \\
a (degrees) & 90 \\
$\beta$ (degrees) & 90 \\
Y (degrees) & 120
\end{tabular}

Chains per asymmetric unit

$1 \mathrm{DHO}, 1 \mathrm{ATC}$

Data collection

$\begin{array}{lc}\text { Resolution }(\AA) & 2.20 \\ \text { Reflections used } & 53365 \\ \text { Completeness (\%) } & 99.4(100.0) \\ \text { Average I/ } \sigma & 16.2(2.3) \\ R_{\text {merge }}(\%) & 10.3(75.6)\end{array}$

Refinement

R factor (\%)

$16.1(24.3)$

$\mathrm{R}$ free (\%)

$20.3(29.3)$

Average B factor (Å2) $\quad 41.3$

Atoms in asymmetric unit $\quad 6151$

Number of water molecules $\quad 445$

Rms bonds ( $\AA$ ) $\quad 0.016$

Rms angle (degree) $\quad 1.558$

$\begin{array}{ll}\text { Rms chiral } & 0.104\end{array}$

center might be exploited to distinguish, for example, between human and some pathogenic DHOs.

To test the possibility that the A. aeolicus DHO may require a second zinc ion, the isolated DHO-ATC complex was titrated with zinc and the catalytic activity was assayed. The observed small increase in catalytic activity could be explained if the $\alpha$-site metal ion occupancy was somewhat less than $100 \%$. This interpretation was confirmed by zinc analysis of the isolated DHO subunit and the DHO-ATC complex, which showed that the occupancy of the isolated DHO subunit from which the dodecamer was reconstituted was $0.84 \mathrm{zinc} / \mathrm{mol}$ of subunit. Similarly, the steady state kinetic parameters were unaltered in the presence of a five-fold molar excess of zinc.

The most convincing evidence demonstrating that a second zinc ion is not required for catalysis was the replacement of two $\beta$-site first shell ligands, His180 and His232, with alanine, which eliminated the $\beta$-site but had no significant effect on the steady state kinetic 


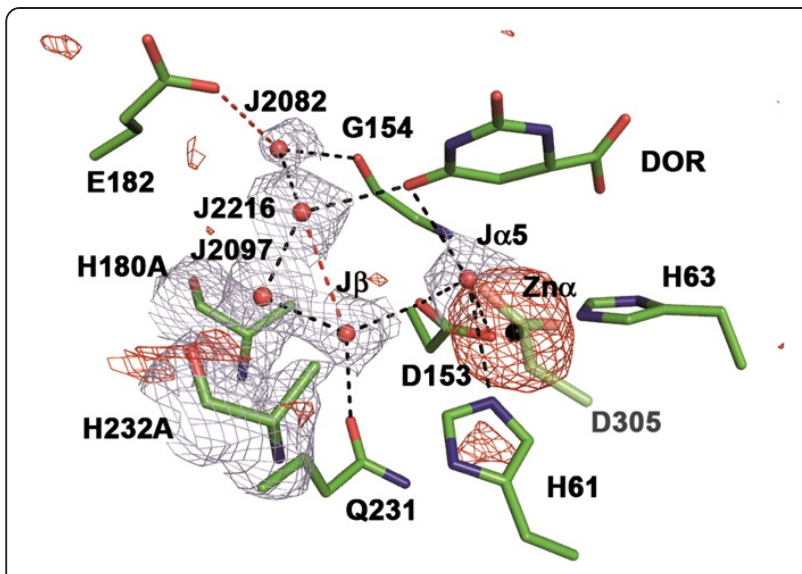

Figure 6 Water structure and anomalous difference density in the active site of the double mutant. The 2Fo-Fc electron density (blue mesh) is shown for the 5 water molecules contoured at 1 sigma and the two mutated alanine residues contoured at 1.5 sigma. When the anomalous difference density map was contoured at 3 sigma for the entire active site, strong difference density was observed only at the a-zinc atom (black sphere, red mesh) with a few noise contours near H61 and H232A. In particular, there was no anomalous difference density at $J \beta$, the putative $\beta$-metal site.

parameters. In contrast, the replacement of the corresponding histidines in the $\beta$ site of Klebsiella pneumoniae dihydroorotase [18] and allantoinase [19], both of which have a binuclear metal site, completely abolished catalytic activity. The crystal structure of the active double mutant showed a zinc atom in the $\alpha$-site and a water molecule $\mathrm{J} \beta$ $(\mathrm{HOH} 2080 / \mathrm{A})$ near the $\beta$-site (Figure 2B, Figure 6) - thus eliminating the unlikely possibility that the one zinc atom was randomly distributed between the two sites. The observation that the elimination of the $\beta$-site ligands of the

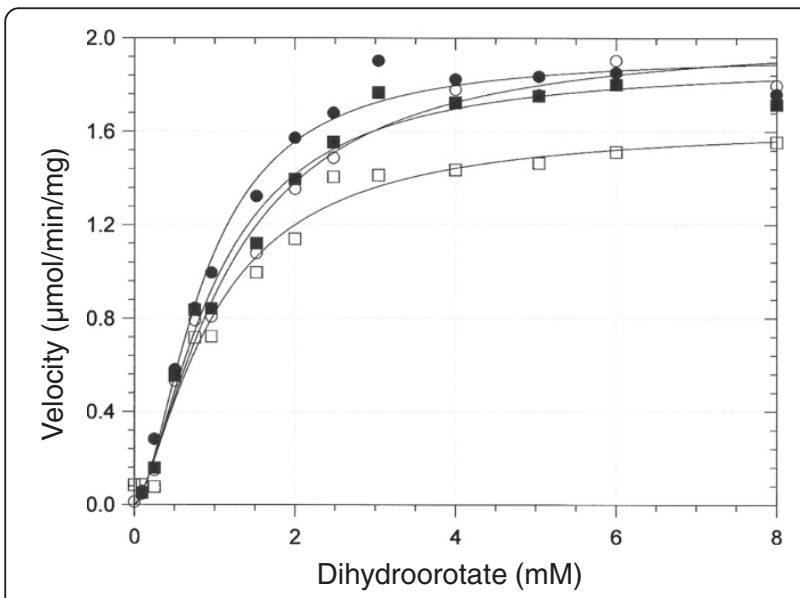

Figure 7 Steady state kinetics of the wild type and $\beta$-site mutants. A dihydroorotate saturation curve of DHO-ATC assayed as described in the Methods ( $250 \mathrm{mM}$ Tris acetate, $\mathrm{pH} 8.3$ at $72^{\circ} \mathrm{C}$ ) was

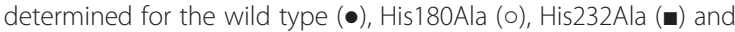
the His180Ala/His232 ( $\square$ ). The kinetic parameters are summarized in Table 4. binuclear metal site enzymes abolishes catalytic activity, whereas there is no effect if the comparable ligands in the mononuclear enzyme are replaced, reinforces the argument that the two types of enzymes are distinct.

An alternate possibility should be considered that perhaps all dihydroorotases utilize a single zinc atom for catalysis and that the second zinc serves another purpose, perhaps enhancing the stability of the protein. This idea is countered by the mutagenesis studies, although it could be argued that the $\beta$-site ligands have an alternate unknown role in catalysis. However, we believe that this hypothesis is unlikely to be true as a plausible two-metal mechanism for E. coli DHO has been proposed based on the structure [3] that is well supported by subsequent biochemical [20] and quantum mechanical studies [21]. We have recently undertaken a molecular dynamics/quantum mechanical investigation based on the structure of the A. aeolicus DHO-ATC complex [12] that suggests that a one-zinc DHO can readily catalyze the reaction by an analogous, albeit somewhat different, mechanism. As discussed below, there is ample precedence for amidohydrolases catalyzing the same reaction having either one or two zinc ions depending on the organism.

\section{Mechanistic considerations}

The structure of the double mutant enzyme offers other insights that are potentially relevant to the mechanism of the native enzyme. First, the catalytic water molecule, which is bound to the two zincs as J $\alpha 5 \beta 5$ in the E. coli enzyme (Figure 2A) and the one zinc as Ja5 in A. aeolicus DHO (1rtf.pdb; Figure 2A) is retained in the mutant structure (J 5 : Figure 2B:, Figure 6), where it is poised, as in the E. coli complex with dihydroorotate (1j79A.pdb), to attack and cleave the ring forming carbamoyl aspartate. In the substrate complex with carbamoyl aspartate (1j79B.pdb, not shown) and the pseudo-substrate complex with citrate (3d6nA.pdb; Figure 2B), an oxygen atom of the ligand occupies the catalytic water site and binds directly to the zinc atom.

A second notable observation is the retention of a water molecule, $\mathrm{HOH} 2080 / \mathrm{A}$ (Jß:, Figure 2B, Figure 6) in the approximate location of the $\mathrm{Zn} \beta$ site in the structure of the mutant DHO despite the elimination of His180 and His232 that hydrogen bonds to the equivalent $\mathrm{J} \beta$ water molecule, HOH577, in the native protein complex (3d6nA. $\mathrm{pdb}$, Figure $2 \mathrm{~B}$ ). In the mutant $\mathrm{DHO}$, the space normally occupied by the imidazole rings of His 180 and His 232 is now occupied, respectively, by $\mathrm{HOH} 77$ and $\mathrm{HOH} 280$, which are also hydrogen bonded with the $\beta$-site water, HOH426 (Figure 6). Extensive theoretical calculations currently underway suggest that this second catalytic water molecule can significantly lower the activation energy of the reaction. 
Although the mutant $A$. aeolicus DHO has alanine replacing the two histidines and dihydroorotate replacing citrate, it is still very similar to the native structure as evidenced by a rmsd value of $0.29 \AA$ for the $422 \mathrm{C} \alpha$ atoms. The two largest $C \alpha$ displacements - Met1 (2.8 $\AA$ ) and Pro41 (1.7 ̊) - occur in the composite domain, which has higher B-values and worse density than the TIM-barrel catalytic domain, for which all the $\mathrm{C} \alpha$ displacements are less than one angstrom. This modest sensitivity to the ligand in the active site contrasts strongly with E. coli DHO, in which a mobile loop (Pro105 - Gly115) is hydrogen bonded to the substrate, carbamoyl aspartate, via residues Thr109 and Thr110 but flipped out of the active site when the product, dihydroorotate, is present (1ekx.pdb) [22]. The $\alpha$-carbons of the two threonines are shifted by approximately $12 \AA$. When the mutant DHO is superposed using SSM onto the E. coli DHO subunit containing dihydroorotate (1ekxA.pdb), Met158 and Asp159 align structurally with Asn107 and Ser118, respectively in the E. coli subunit. In other words, SSM identifies the mobile loop in E. coli $\mathrm{DHO}$, as a ten residue insert compared to the structure of $A$. aeolicus DHO. Interestingly, when the mutant $\mathrm{DHO}$ structure is superposed onto the E. coli DHO subunit containing carbamoyl aspartate (1ekxB.pdb), there are no $A$. aeolicus residues equivalent to Thr109 and Thr110 to hydrogen bond to the substrate. However, this observation must be verified by determining the structure of $A$. aeolicus DHO with the carbamoyl aspartate substrate in its active site.

\section{ATC conformational changes induced by PALA}

The mutant DHO-ATC structure is the first with the bisubstrate analog, N-phosphoacetyl L-aspartate (PALA) bound to the active site of the $A$. aeolicus ATC subunit (Figure 5B). The PALA-ATC subunit superposes onto the native structure with an rmsd value of $0.71 \AA$ for 291 residues and exhibits two major conformational changes triggered by PALA replacing citrate in the active site. The residues from Ser67 to Glu77 have $\mathrm{C} \alpha$ displacements above $1 \AA$ with a maximum of $7.4 \AA$ at Ser72. Together, Ser72 and Lys75 in this segment form hydrogen bonds five in the mutant structure - directly to the PALA in the adjacent catalytic subunit, as first reported for E. coli ATC [23]. The second major conformational change involves segment Arg213 through Asn222, which has a maximum C $\alpha$ displacement of $8.5 \AA$ at Gln219. Arg213 and Gln215 form hydrogen bonds directly with the C5 carboxyl group of PALA. The large displacement of Gln219, which has no significant interactions with PALA or other residues, is a consequence, and not a cause, of the conformational change. An adjacent residue, Arg218, which has a $\mathrm{C} \alpha$ displacement of $7.8 \AA$, interacts indirectly with PALA through a hydrogen bond network with the PALA ligands Gln215, and Arg159.
In $E$. coli ATC, PALA binding triggers the transition from the $\mathrm{T}$ state to the active $\mathrm{R}$ state. Although the two largest conformational changes induced by PALA are "homologous" in the A. aeolicus and E. coli ATC, the former is much less plastic. The mutant $A$. aeolicus ATC has only 27 out of 291 residues with a $\mathrm{C} \alpha$ displacement greater than $1.0 \AA$ relative to the native structure whereas the $E$. coli enzyme has 109 out of 310 residues that move over $1.0 \AA$ due to PALA binding. This difference in conformational flexibility might be due to the native $A$. aeolicus structure having citrate bound in the PALA site, so it is already partially in the R-state, or to the A. aeolicus ATC being part of a larger complex that inhibits widespread conformational changes.

E. coli ATC, which is a complex of two trimers of catalytic chains and three dimers of regulatory chains, is notable for its large quaternary changes associated with the $\mathrm{T}$ to $\mathrm{R}$ transition upon PALA binding. The hetero dodecamer expands by $12 \AA$ and the two trimers rotate by 10 degrees relative to each other [23]. However, PALA binding to the A. aeolicus mutant dodecamer, which is not an allosteric enzyme, has only a very modest effect. The "top" and "bottom" ATC trimers in the mutant dodecamer generated from crystallographic symmetry, when compared to the native complex, have rotated less than 2 degrees relative to each other and expanded by less than $0.5 \AA$.

\section{Comparison of amidohydrolase mono and dinuclear metal centers}

Sequence alignment (Figure 8) shows that the one-zinc DHOs from $A$. aeolicus and $S$. aureus exhibit the highest sequence similarity (41\% identity and $59 \%$ similarity) to each other. The A. aeolicus enzyme is much less similar to either of the two zinc enzymes, E. coli (23\% identity, $39 \%$ similarity) or the mammalian enzyme $(30 \%$ identity, $46 \%$ similarity). The differences in sequence per se cannot explain the differences between the one and two zinc enzymes, but suggest a closer phylogenetic relationship between the enzymes that bind the same number of metal ions.

Although E. coli $\mathrm{DHO}$ and all other type-II DHO structures have two zinc atoms in their active sites, the type-I DHO structures vary among themselves. All of the first and second shell zinc ligands of all type I enzymes of known structure, match those of $A$. aeolicus $\mathrm{DHO}$ in type and position, but only the $S$. aureus enzyme (3gri.pdb) has an empty $\beta$-site like the $A$. aeolicus DHO. In contrast, the enzymes from B. anthracis [24] (3mpg/pdb) and T. thermophilus (2z00.pdb) have two active site zinc atoms, although the occupancy of the $\beta$ site of the latter enzyme is only 0.4 . The residues in two ligation shells around the zinc atoms in $A$. aeolicus $\mathrm{DHO}$ and the three other type-I DHOs were compared 


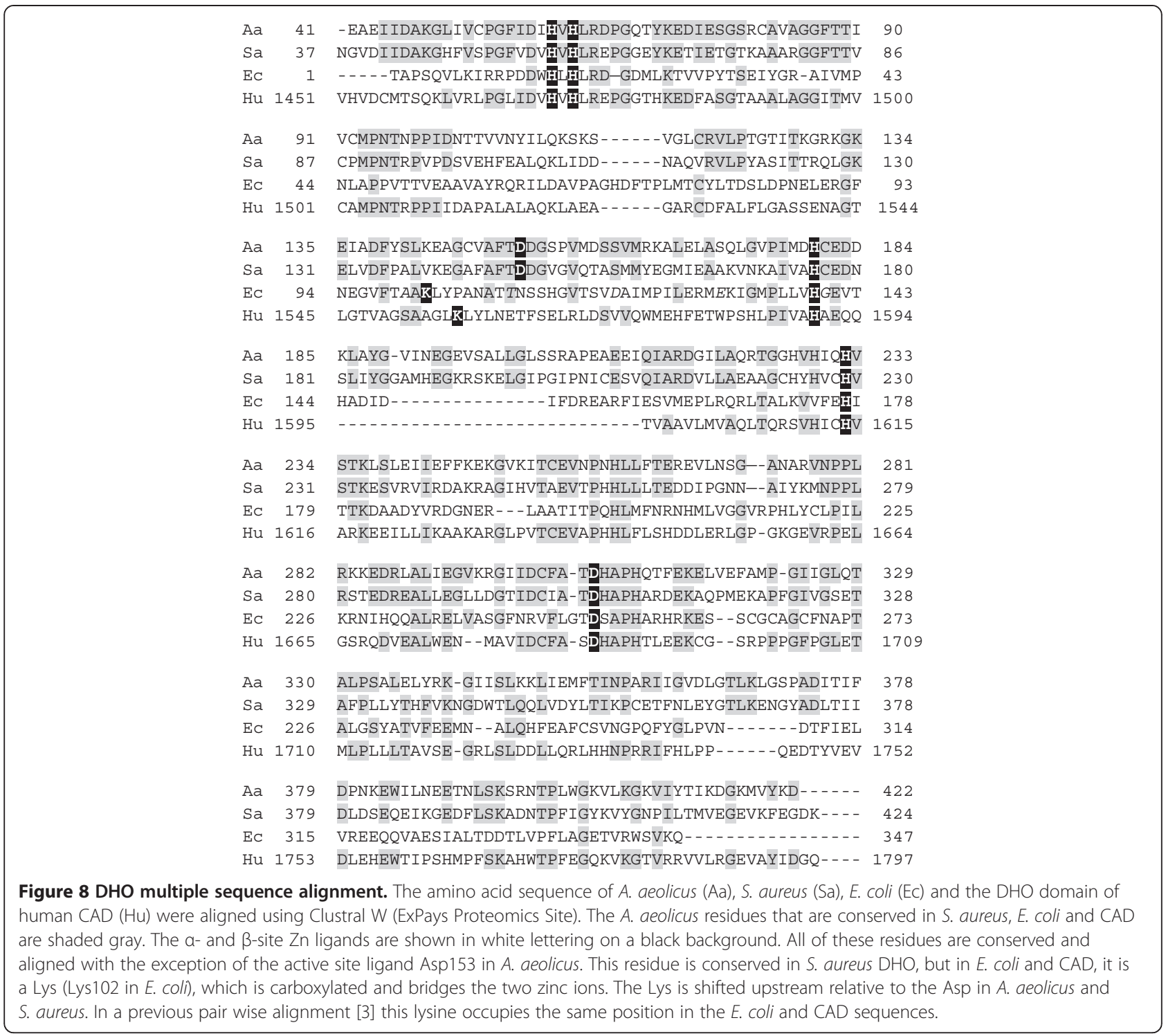

to understand how the metal centers of these enzymes differ (Figure 2C; see Additional file 1: Table S1).

There is precedence for variation in the metal content of the same enzymes in the amidohydrolyase superfamily. $N$-acetyl-D-galactosamine-6-phosphate deacetylase (NagA) [25] from Bacillus subtilis binds two metals, whereas NagA from Escherichia coli and Thermotoga maritima bind a single metal ion at the $\beta$-site. E. coli NagA lacks the $\mathrm{HxH}$ motif that forms the $\alpha-\mathrm{Zn}$ site in $B$. subtilis NagA, all known type-I and type-II DHOs and many other amidohydrolases. This signature sequence is replaced by QXN and the resulting weaker metal affinity may explain why this enzyme only binds a single metal ion at the $\beta$-site [25]. A phylogenetic analysis [25] of all the NagA homologs revealed that a histidine residue located in the second ligand shell of the $\beta$-site (corresponding to His-143 in E. coli
NagA) is conserved in all of the enzymes that cluster with the one zinc enzymes. In the two metal enzyme cluster, this residue is replaced with Glu or Gln. The authors suggest that the histidine may polarize the carbonyl bond of the substrate during hydrolysis, in effect compensating for the missing second metal ion.

However, this difference between the mono- and dinuclear enzymes is not found among the dihydroorotases. The four type-I dihydroorotases, both mono- and dinuclear, have a glycine residue (Gly154 for A. aeolicus) filling the space of the polarizing histidine imidazole ring, while the E. coli type-II DHO, has empty space. Moreover, the only sequence difference in the $\mathrm{Zn}$ ligation shells among the four type-I DHO structures, which occurs at position $\beta 5$ in Figure $2 \mathrm{C}$, does not correlate with zinc binding (Additional file 1: Table S1). The $A$. aeolicus (one zinc) 
and T. thermophilus (two zincs) enzymes have a glutamine residue at this location while $S$. aureus (one zinc) and B. anthracis (two zincs) enzymes have a cysteine residue.

Sequence differences in the second shell are another common explanation for variable metal affinity of histidinerich sites. A thermodynamic analysis of zinc binding of human carbonic anhydrase provided evidence that the second shell residues, in addition to orienting the direct ligands, can also appreciably affect the enthalpy of metal binding [26]. Mutation of the second shell ligands Gln92, Glu117, and Thr199 reduces the affinity of zinc binding 10-fold $[27,28]$. The replacement of Glu117, which normally forms a hydrogen bond with His94 in the first shell, results in a large increase in enthalpy associated with zinc binding [27]. The structure of this mutant [28] revealed that a halide ion occupies the void created by the missing Glu side chain, but otherwise the structure of the active site is not significantly different than the wild type enzyme $\left(C_{\alpha}\right.$ rmsd $\left.=0.2 \AA\right)$.

Aspartate or glutamate residues can act as proton acceptors for the histidine residues in the first shell and thereby strengthen their interaction with the zinc atom $[29,30]$. This interaction is weaker when amides occupy the second shell position. However, with the type-I DHO structures, the relevant second shell ligands are the same for all four type-I DHO structures - a glutamate in $\alpha 1^{\prime}$, an asparagine in $\alpha 2^{\prime}$, a glutamate in $\beta 1^{\prime}$ and water molecules in $\beta 2^{\prime}$ (Figure 2C, Additional file 1: Table S1). Looking deeper, the $\beta 1$ ' glutamic acid is significantly farther from the $\beta 1$ histidine in both $A$. aeolicus $(3.3 \AA$ ) and S. aureus DHO (4.5 $\AA$ ) - implying weaker zinc binding - than in T. thermophilis DHO (2.8 $\AA$ ). However, the distance is just as long in B. anthracis DHO (3.4 $\AA$ ), so again there is no simple correlation between the geometry and the relative metal affinity.

Consequently, a complete explanation of this difference in zinc affinities of the $\mathrm{Zn} \beta$ sites in the two proteins is beyond the purview of this study. For example, a recent analysis of two carbonic anhydrases had to include all atoms within $9 \AA$ of the zinc atom in quantum mechanics calculations to fully explain the relative zinc affinities and catalytic properties [31,32].

\section{Conclusions}

Taken together, these results indicate that although an apparent viable $\beta$-zinc binding site is formed when the A. aeolicus DHO domain associates tightly with ATC presumably the natural state of these enzymes - the data presented here indicate that additional zinc does not bind to the enzyme or influence the catalytic activity. We therefore conclude that $A$. aeolicus DHO-ATC complex does not require a second metal for catalysis, although the conservation of the second site during evolution leaves open the possibility that a second ion might bind in vivo.
However, here we have shown that this type-I DHO is fully functional with a mononuclear metal center.

\section{Methods \\ Materials}

All chemicals were purchased from Sigma-Aldrich. $E$. coli strains DH5 $\alpha$ and BL21 (DE3) were from Invitrogen.

\section{Isolation of the DHO and ATC subunits and formation of the DHO-ATC complex}

The genes encoding $A$. aeolicus DHO (pyrC) and ATC (pyrB) subunits were previously cloned and separately expressed in $E$. coli $[5,6,33]$. The genes were identified in the A. aeolicus genome [34], amplified by PCR and inserted into pRSETC (Invitrogen), an expression vector that incorporates a $3 \mathrm{kDa}$ His tag on the amino end of the recombinant protein. The proteins were expressed individually in BL21 (DE3) and purified by $\mathrm{Ni}^{+2}$ affinity chromatography on a $1-\mathrm{ml} \mathrm{Ni}^{+2}$ Probond column (Invitrogen). While the DHO from some organisms has been found to have limited thermal stability $[4,19]$, the DHO and ATC from $A$. aeolicus, a hyperthermophile that grows at $95^{\circ} \mathrm{C}$ [34], are stable for several months when stored in $50 \mathrm{mM}$ TrisHCl, $200 \mathrm{mM} \mathrm{NaCl}, 200-300 \mathrm{mM}$ imidazole, $\mathrm{pH} 8.0$ at $4^{\circ} \mathrm{C}$.

The purified subunits were mixed in an equimolar ratio to reconstitute the dodecameric DHO-ATC complex $[6,12]$. The complex was isolated by gel filtration chromatography on a Sephacryl S-300 column. The composition of the complex was analyzed by SDS polyacrylamide gel electrophoresis [35] and ATC and DHO assays. For crystallization, the complex was concentrated to $3 \mathrm{mg} / \mathrm{ml}$ using centrifugal filters (Amicon Ultra) with a $10 \mathrm{kDa}$ molecular weight cut off. Protein concentrations were determined by the Lowry method [36] using bovine serum albumin as a standard.

\section{Enzyme assays}

The ATC activity was measured by the colorimetric method previously described $[37,38]$. The reaction contained $2 \mathrm{mM}$ aspartate, $5 \mathrm{mM}$ carbamoyl phosphate, $1-$ $11 \mu \mathrm{g}$ of the purified enzyme, and $50 \mathrm{mM}$ Tris- $\mathrm{HCl}, \mathrm{pH}$ 8 , in a total volume of $0.5 \mathrm{ml}$. The incubation time was $10 \mathrm{~min}$ at $37^{\circ} \mathrm{C}$ or $1 \mathrm{~min}$ at $75^{\circ} \mathrm{C}$. The reactions were quenched by the addition of an equal volume of $5 \%$ acetic acid. The color was developed and quantitated as described previously [33].

DHO activity was measured in the reverse direction, the formation of carbamoyl aspartate from dihydroorotate, because the equilibrium strongly favors dihydroorotate hydrolysis [39] at pH 8.3. The formation of carbamoyl aspartate was measured using the same colorimetric method. The assay mixture, consisting of 20-45 $\mu \mathrm{g}$ DHO in 50 $\mathrm{mM}$ Tris acetate, $\mathrm{pH} 8.3,10 \%$ glycerol, was preincubated 
for $1.5 \mathrm{~min}$ at $75^{\circ} \mathrm{C}$. The reaction was initiated by the addition of dihydroorotate ( $8 \mathrm{mM}$ or variable) and quenched after 1.5-5 min [6]. The kinetic parameters were obtained by least squares analysis of the dihydroorotate saturation curves using the program KaleidaGraph.

\section{Site-directed mutagenesis}

Site-directed mutagenesis was carried out by PCR using Pfu Turbo polymerase (Stratagene) and the plasmid encoding the DHO subunit as a template. The putative $\beta$ site zinc ligands, His180 and His232, were replaced with alanine using forward and reverse oligonucleotides from Invitrogen. The fidelity of the constructs was verified by sequencing.

\section{Preparation of the metal free Apo-enzyme}

Wild type DHO (25 mg) was dialyzed against $250 \mathrm{ml}$ of $50 \mathrm{mM}$ sodium MES, $25 \mathrm{mM}$ pyridine-2,6-dicarboxylate, $1 \mathrm{mM}$ EDTA, 30\% glycerol (v/v), $50 \mathrm{mM}$ sodium sulfate, $0.5 \mathrm{mM}$ DTT, pH 5.5, with two changes of the dialysis buffer over three days [6]. The apoenzyme was concentrated and desalted by passage over a Sephadex G-25 column equilibrated with $20 \mathrm{mM}$ MES, $20 \%$ glycerol $0.1 \mathrm{mM}$ TCEP, $\mathrm{pH}$ 6.3. The protein was then concentrated to $10 \mathrm{mg} / \mathrm{ml}$. The complete removal of zinc was verified by zinc spectrophotometric analysis and the DHO-ATC complex was reconstituted by mixing equimolar amounts of the metal-free DHO and ATC subunits.

\section{Metal analysis}

The zinc content was measured by determining the absorbance of the complex of zinc with 4-(2-pyridylazo)resorcinol (PAR), in $50 \mathrm{mM}$ HEPES, pH 7.4 [40]. The DHO-ATC $(10 \mu \mathrm{M})$ was denatured in $4 \mathrm{M}$ guanidine $\mathrm{HCl}$ for $15 \mathrm{mi}-$ nutes and PAR was added to a final concentration of $50 \mu \mathrm{M}$. The absorbance was read at $497 \mathrm{~nm}$, the $\lambda_{\max }$ of Zn-PAR $\left(\varepsilon_{497}=46,700 \mathrm{M}^{-1} \mathrm{~cm}^{-1}\right)$. The zinc released from the protein was calculated using a $\mathrm{ZnCl}_{2}$ standard curve $(0-20 \mu \mathrm{M})$. Alternatively, the zinc and cobalt composition of the protein was analyzed by inductively coupled plasma (ICP) mass spectrometry. The purified proteins in $50 \mathrm{mM}$ TrisHCl, $200 \mathrm{mM} \mathrm{NaCl}$ and $2 \mathrm{mM}$ DTT were supplemented where indicated with $100 \mu \mathrm{M} \mathrm{ZnCl}_{2}$. The excess zinc was removed by gel filtration on a G25 column in the same buffer. The final protein concentration was approximately $40 \mu \mathrm{M}$ as determined by the Lowry method [36]. The samples were acidified by the addition of $\mathrm{HNO}_{3}$ to 2\% and analyzed in a Perkin Elmer Sciex Elan 9000 ICPMS with a cross flow nebulizer and Scott type spray chamber. The RF power was 1000 and the argon flow was set at $0.92 \mathrm{~L} / \mathrm{min}$. Analytical grade standard metal stock solutions were purchased from Sigma-Aldrich and VWR.

\section{Crystallization of the DHO-ATC mutant}

Before crystallization, the protein was buffer exchanged into $10 \mathrm{mM}$ HEPES, $1 \mathrm{mM}$ TCEP, $\mathrm{pH}$ 7.5, at a final protein concentration of $3.0 \mathrm{mg} / \mathrm{ml}$. All crystals were grown at room temperature using the hanging-drop, vapordiffusion technique: 3-6 $\mu$ l of the DHO-ATC solution was mixed with $1 \mu \mathrm{l}$ of reservoir solution (30\% ethylene glycol) followed by $10 \%$ of the drop volume (v/v) of 100 $\mathrm{mM}$ barium chloride as a crystallization additive [12]. Prism-like crystals appeared within 2-7 days; the best diffracting crystals grew to $0.2 \times 0.2 \times 0.1 \mathrm{~mm}$ and were often accompanied by small needle-like crystals. Prior to data collection, the crystals were soaked overnight in the cryo solution: $10 \mathrm{mM} \mathrm{BaCl}, 35 \% \mathrm{v} / \mathrm{v}$ ethylene glycol, 1 mM PALA and $15 \mathrm{mM}$ dihydroorotate at $\mathrm{pH} 5$.

\section{Phasing and refinement of the DHO-ATC double mutant}

The X-ray diffraction data were collected in 360 frames at the Advanced Photon Source (IMCA-CAT) using a wavelength of $0.97856 \AA$ and a rotation of 0.75 degree per frame. The data were integrated with iMosfilm [41] and truncated at $2.2 \AA$ where the average intensity in the highest shell was approximately twice the estimated error. The initial electron density map was calculated using phases of the native DHO-ATC [12], refit with ARP/wARP [42] to reduce bias, and refined with REFMAC5 [43]. All solvent density was initially fit and refined with water molecules. Strong solvent peaks with anomalous diffraction density were refit with barium ions. Clusters of 4 water molecules at unusually short distances from each other were refit with molecules of ethylene glycol. The final ratio of $R_{\text {free }} / R$ was 1.26 , which is very close to the expected value of 1.25 at this resolution [44]. There are no residues in the disallowed regions of the Ramachandran plot calculated by PROCHECK.

\section{Modeling and analysis}

Unless stated otherwise, structures compared in this paper were superposed with SSM [45], which includes secondary structure matches in the calculation. SPDBV [46] was used to overlap structures based upon specific residues. To facilitate comparison of the active sites, a virtual second zinc site (Zn1006) was modeled into the structure of $S$. aureus DHO (3gri.pdb; Zn500) by overlapping the DHO structure from T. thermophilis (2z00.pdb; Zn1004, Zn1006) using only the main chain atoms of the $\alpha 1, \alpha 2$, and $\alpha \beta 4$ residues (Figure 2C). This approach placed the respective $\mathrm{Zn} \alpha$ atoms (Zn500, Zn1004) within $0.2 \AA$ of each other and the transformed $\mathrm{Zn} \beta$ atom ( $\mathrm{Zn1006)} 3.2 \AA$ from the native $\mathrm{Zn} \alpha$ atom (Zn500) in the $S$. aureus structure. Panels showing crystallographic structures were prepared with Pymol [47]. 


\section{Availability of supporting data}

The data set supporting the results of this article is included in the article and its Additional file 1: Table S1.

\section{Endnotes}

${ }^{\text {a }}$ The structure of mammalian $\mathrm{DHO}$ was presented by Santiago Ramon-Maiques at the 23rd International Conference on Arginine and Pyrimidines held at the Universidad de los Andes, Bogotá, Colombia. The manuscript is pending publication. A preliminary X-ray analysis of the crystals has been published [48].

${ }^{b}$ The atomic coordinates for the crystal structure discussed herein will be available from the Research Collaboratory for Structural Bioinformatics with accession code $4 \mathrm{BJH}$ upon publication.

\section{Additional file}

Additional file 1: Table S1. Interaction parameters for the first and second ligation shells of Zna and Zn $\beta$ depicted in Figure 1C.

\section{Abbreviations}

AQUAE: Aquifex aeolicus; ATC: Aspartate transcarbamoylase; BACAN: Bacillus anthracis; CAD: A polypeptide chain present in mammals and some other species that includes the first three enzymes of pyrimidine biosynthesis fused in the order, CPS-DHO-ATC; CPS: Carbamoyl phosphate synthetase; DAC: Dihydroorotaseaspartate transcarbamoylase complex; DHO: Dihydrorotase; DOR: Dihydroorotate; PALA or PALN: (Phosphonacetyl)-L-aspartate; PAR: 4-(2-pyridylazo)resorcinol; STACC: Staphylococcus aureus; THETA8: Thermus thermophilus.

\section{Competing interests}

The authors declare that they have no competing interests.

\section{Author's contributions}

RF, EG and MC carried out the metal analysis and kinetic studies; RF also constructed the DHO mutants; $\mathrm{AV}$ isolated the proteins, reconstituted the $\mathrm{DHO}$ ATC complex, conducted the ICP metal analysis and grew the protein crystals; JB collected the X-ray crystallography data and carried out the initial analysis of the data; PM solved the structure of the mutant complex; BFPE refined and analyzed the final, submitted structure; BFPE, HE and DRE were primarily responsible for the experimental design, interpretation of the data and writing the manuscript. All authors read and approved of the final manuscript.

\section{Acknowledgements}

${ }^{\dagger}$ This work was supported in part by NIH Grants HL57527, HL/GM47399, and GM/CA60371, NSF Grant MCB9810325, and a grant for computational biology from Wayne State University. The Advanced Laboratory for Macromolecular Crystallography at Wayne State University was supported by Grant 085P1000817 from the Michigan Economic Development Corporation and Technology Tri-Corridor. Use of the Advanced Photon Source was supported by the U.S. Department of Energy, Basic Energy Sciences, Office of Science, under Contract W-31-109-Eng-38. Use of the Advanced Photon Source, an Office of Science User Facility operated for the U.S. Department of Energy (DOE) Office of Science by Argonne National Laboratory, was supported by the U. S. DOE under Contract No. DE-AC02-06CH11357. Use of the LS-CAT Sector 21 was supported by the Michigan Economic Development Corporation and the Michigan Technology Tri-Corridor (Grant 085P1000817). We wish to thank Ashoka Kandegedara (Wayne State University) for running the ICP analyses.

\section{Author details}

${ }^{1}$ Department of Biochemistry and Molecular Biology, Wayne State University School of Medicine, 540 East Canfield Street, Detroit, MI 48201, USA. ${ }^{2}$ Department of Chemistry, Eastern Michigan University, Ypsilanti, MI 48197, USA. ${ }^{3}$ Life Sciences Collaborative Access Team, Northwestern University Center for Synchrotron Research, Argonne, IL 60439, USA.
Received: 18 August 2013 Accepted: 28 October 2013

Published: 9 December 2013

\section{References}

1. Holm L, Sander C: An evolutionary treasure: unification of a broad set of amidohydrolases related to urease. Proteins 1997, 28:72-82.

2. Fields C, Brichta D, Shephardson M, Farinha M, O'Donovan G: Phlyogenetic analysis and classification of dihydroorotases: a complex history for a complex enzyme. Paths Pyrimidines 1999, 7:49-63.

3. Thoden JB, Phillips GN Jr, Neal TM, Raushel FM, Holden HM: Molecular structure of dihydroorotase: a paradigm for catalysis through the Use of a binuclear metal center. Biochemistry 2001, 40:6989-6997.

4. Washabaugh MW, Collins KD: Dihydroorotase from Escherichia coli. Purification and characterization. J Biol Chem 1984, 259:3293-3298.

5. Purcarea C, Martin P, Vickrey JF, Guy HI, Edwards BF, Evans DR: Cloning, expression and preliminary $\mathrm{X}$-ray analysis of the dihydroorotase from the hyperthermophilic eubacterium aquifex aeolicus. Acta Crystallogr D Biol Crystallogr 2002, 58:154-156.

6. Ahuja A, Purcarea C, Ebert R, Sadecki S, Guy HI, Evans DR: Aquifex aeolicus dihydroorotase: association with aspartate transcarbamoylase switches on catalytic activity. J Biol Chem 2004, 279:53136-53144. Epub 52004 Sep 53120.

7. Martin PD, Purcarea C, Zhang P, Vaishnav A, Sadecki S, Guy-Evans HI, Evans $\mathrm{DR}$, Edwards BF: The crystal structure of a novel, latent dihydroorotase from aquifex aeolicus at 1.7A Resolution. $J$ Mol Biol 2005, 348:535-547.

8. Ireton GC, McDermott G, Black ME, Stoddard BL: The structure of Escherichia coli cytosine deaminase. J Mol Biol 2002, 315:687-697.

9. Davies BJ, de Vries N, Rijpkema SG, van Vliet AH, Penn CW: Transcriptional and mutational analysis of the helicobacter pylori urease promoter. FEMS Microbiol Lett 2002, 213:27-32

10. $X u Z$ Z, Liu Y, Yang Y, Jiang W, Arnold E, Ding J: Crystal structure of D-hydantoinase from burkholderia pickettii at a resolution of 2.7 Angstroms: insights into the molecular basis of enzyme thermostability. J Bacteriol 2003, 185:4038-4049.

11. Vincent F, Yates D, Garman E, Davies GJ, Brannigan JA: The three-dimensional structure of the $\mathrm{N}$-acetylglucosamine-6-phosphate deacetylase, NagA, from bacillus subtilis: a member of the urease superfamily. J Biol Chem 2004, 279:2809-2816.

12. Zhang P, Martin PD, Purcarea C, Vaishnav A, Brunzelle JS, Fernando R, Guy-Evans HI, Evans DR, Edwards BF: Dihydroorotase from the hyperthermophile aquifex aeolicus is activated by stoichiometric association with aspartate transcarbamoylase and forms a one-pot reactor for pyrimidine biosynthesis. Biochemistry 2009, 48:766-778.

13. Evans DR, Guy HI: Mammalian pyrimidine biosynthesis: fresh insights into an ancient pathway. J Biol Chem 2004, 279:33035-33038.

14. Zimmermann BH, Evans DR: Cloning, overexpression, and characterization of the functional dihydroorotase domain of the mammalian multifunctional protein CAD. Biochemistry 1993, 32:1519-1527.

15. Kelly RE, Mally MI, Evans DR: The dihydroorotase domain of the multifunctional protein CAD. Subunit structure, zinc content, and kinetics. J Biol Chem 1986, 261:6073-6083.

16. Zimmermann BH, Kemling NM, Evans DR: Function of conserved histidine residues in mammalian dihydroorotase. Biochemistry 1995, 34:7038-7046.

17. Huang DT, Thomas MA, Christopherson RI: Divalent metal derivatives of the hamster dihydroorotase domain. Biochemistry 1999, 38:9964-9970.

18. Wang CC, Tsau HW, Chen WT, Huang CY: Identification and characterization of a putative dihydroorotase, KPN01074, from klebsiella pneumoniae. Protein J 2010, 29:445-452.

19. Ho YY, Huang $Y H$, Huang $C Y$ : Chemical rescue of the post-translationally carboxylated lysine mutant of allantoinase and dihydroorotase by metal ions and short-chain carboxylic acids. Amino Acids 2013, 44:1181-1191.

20. Porter TN, Li Y, Raushel FM: Mechanism of the dihydroorotase reaction. Biochemistry 2004, 43:16285-16292.

21. Liao RZ, Yu JG, Raushel FM, Himo F: Theoretical investigation of the reaction mechanism of the dinuclear zinc enzyme dihydroorotase. Chemistry 2008, 14:4287-4292.

22. Lee M, Chan CW, Graham SC, Christopherson RI, Guss JM, Maher MJ: Structures of ligand-free and inhibitor complexes of dihydroorotase from Escherichia coli: implications for loop movement in inhibitor design. J Mol Biol 2007, 370:812-825. 
23. Krause KL, Volz KW, Lipscomb WN: 2.5 A structure of aspartate carbamoyltransferase complexed with the bisubstrate analog $\mathrm{N}$-(phosphonacetyl)-L-aspartate. J Mol Biol 1987, 193:527-553.

24. Mehboob S, Mulhearn DC, Truong K, Johnson ME, Santarsiero BD: Structure of dihydroorotase from bacillus anthracis at 2.6 A resolution. Acta Crystallogr Sect F: Struct Biol Cryst Commun 2002, 66:1432-1435.

25. Hall RS, Brown S, Fedorov AA, Fedorov EV, Xu C, Babbitt PC, Almo SC, Raushel FM: Structural diversity within the mononuclear and binuclear active sites of N-acetyl-D-glucosamine-6-phosphate deacetylase. Biochemistry 2007, 46:7953-7962.

26. DiTusa CA, McCall KA, Christensen T, Mahapatro M, Fierke CA, Toone EJ: Thermodynamics of metal ion binding. 2. Metal ion binding by carbonic anhydrase variants. Biochemistry 2001, 40:5345-5351.

27. Kiefer LL, Krebs JF, Paterno SA, Fierke CA: Engineering a cysteine ligand into the zinc binding site of human carbonic anhydrase II. Biochemistry 1993, 32:9896-9900.

28. Kiefer LL, Paterno SA, Fierke CA: Hydrogen bond network in the metal binding site of carbonic anhydrase enhances zinc affinity and catalytic efficiency. J Am Chem Soc 1995, 117:6831-6837.

29. Lesburg CA, Christianson DW: X-ray crystallographic studies of engineered hydrogen bond networks in a protein-zinc binding site. J Am Chem SoC 1995, 117:6838-6844.

30. Lin $Y L$, Lim C: Factors governing the protonation state of $\mathrm{Zn}$-bound histidine in proteins: a DFT/CDM study. J Am Chem Soc 2004, 126:2602-2612.

31. Dudev T, Lim C: Metal binding affinity and selectivity in metalloproteins: insights from computational studies. Annu Rev Biophys 2008, 37:97-116.

32. Pannetier F, Ohanessian G, Frison G: Comparison between alpha- and beta-carbonic anhydrases: can $\mathrm{Zn}(\mathrm{His}) 3(\mathrm{H} 2 \mathrm{O})$ and $\mathrm{Zn}(\mathrm{His})(\mathrm{Cys}) 2(\mathrm{H} 2 \mathrm{O})$ sites lead to equivalent enzymes? Dalton Trans 2011, 40:2696-2698.

33. Purcarea C, Ahuja A, Lu T, Kovari L, Guy HI, Evans DR: Aquifex aeolicus aspartate transcarbamoylase, an enzyme specialized for the efficient utilization of unstable carbamoyl phosphate at elevated temperature. J Biol Chem 2003, 278:52924-52934

34. Deckert G, Warren PV, Gaasterland T, Young WG, Lenox AL, Graham DE, Overbeek R, Snead MA, Keller M, Aujay M, Huber R, Feldman RA, Short JM, Olsen GJ, Swanson RV: The complete genome of the hyperthermophilic bacterium aquifex aeolicus. Nature 1998, 392:353-358.

35. Laemmli $U$ : Cleavage of structural proteins during the assembly of the head of bacteriophage T4. Nature 1970, 227:680-685.

36. Lowry O, Rosenbrough N, Farr A, Randall R: Protein measurement with the folin phenol reagent. J Biol Chem 1951, 193:265-275.

37. Prescott LM, Jones ME: Modified methods for the determination of carbamyl aspartate. Anal Biochem 1969, 32:408-419.

38. Pastra-Landis SC, Foote J, Kantrowitz ER: An improved colorimetric assay for aspartate and ornithine transcarbamylases. Anal Biochem 1981, 118:358-363.

39. Christopherson $\mathrm{Rl}$, Jones ME: The effects of $\mathrm{pH}$ and inhibitors upon the catalytic activity of the dihydroorotase of multienzymatic protein pyr1-3 from mouse Ehrlich ascites carcinoma. J Biol Chem 1980, 255:3358-3370.

40. Sabel CE, Shepherd JL, Siemann S: A direct spectrophotometric method for the simultaneous determination of zinc and cobalt in metalloproteins using 4-(2-pyridylazo)resorcinol. Anal Biochem 2009, 391:74-76.

41. Leslie AGW, Powell HR: Evolving methods for macromolecular crystallography. Nato Sci Ser 2007, 245:41-51.

42. Winn MD: An overview of the CCP4 project in protein crystallography: an example of a collaborative project. J Synchrotron Radiat 2003, 10:23-25.

43. Murshudov GN, Skubak P, Lebedev AA, Pannu NS, Steiner RA, Nicholls RA, Winn MD, Long F, Vagin AA: REFMAC5 For the refinement of macromolecular crystal structures. Acta Crystallogr D Biol Crystallogr 2011, 67:355-367.

44. Tickle IJ, Laskowski RA, Moss DS: Rfree and the rfree ratio. I. Derivation of expected values of cross-validation residuals used in macromolecular least-squares refinement. Acta Crystallogr D Biol Crystallogr 1998, 54:547-557.

45. Krissinel E, Henrick K: Secondary-structure matching (SSM), a new tool for fast protein structure alignment in three dimensions. Acta Crystallogr D Biol Crystallogr 2004, 60:2256-2268.

46. Guex N, Peitsch MC: SWISS-MODEL and the swiss-PdbViewer: an environment for comparative protein modeling. Electrophoresis 1997, 18:2714-2723.
47. DeLano WL: The PYMOL molecular graphics system, Currently supported by Schrödinger, L., Ed. DeLano Scientific: Palo Alto, CA; 2002.

48. Lallous N, Grande-Garcia A, Molina R, Ramon-Maiques S: Expression, purification, crystallization and preliminary X-ray diffraction analysis of the dihydroorotase domain of human CAD. Acta Crystallogr Sect F: Struct Biol Cryst Commun 2012, 68:1341-1345.

doi:10.1186/1471-2091-14-36

Cite this article as: Edwards et al:: The mononuclear metal center of type-I dihydroorotase from aquifex aeolicus. BMC Biochemistry 2013 14:36.

\section{Submit your next manuscript to BioMed Central and take full advantage of:}

- Convenient online submission

- Thorough peer review

- No space constraints or color figure charges

- Immediate publication on acceptance

- Inclusion in PubMed, CAS, Scopus and Google Scholar

- Research which is freely available for redistribution 